Enferm Bras 2020;19(3):238-45

https://doi.org/10.33233/eb.v19i3.3948

\title{
ARTIGO ORIGINAL \\ Infecções prevalentes na unidade de terapia intensiva de um hospital universitário
}

Paloma Cavalcante de Assis Martins*, Ana Katly Martins Gualberto Vaz, M.Sc. **

*Enfermeira Residente do Programa Atenção ao Paciente Adulto Neurocirúrgico em UTI pelo Hospital Universitário Getúlio Vargas, Universidade Federal do Amazonas, Bacharel em Enfermagem pela Universidade Federal do Amazonas, **Doutoranda no Programa de PósGraduação em Enfermagem na Saúde do Adulto da Escola de Enfermagem da Universidade de São Paulo, Professora Assistente do Curso de Enfermagem da Universidade Federal do Amazonas

Recebido em 23 de fevereiro de 2020; aceito em 2 de maio de 2020.

Correspondência: Paloma Cavalcante de Assis Martins, Avenida Apurinã, 4, Praça 14 de Janeiro 69020-170 Manaus AM

Paloma Cavalcante de Assis Martins: palomassis@yahoo.com.br

Ana Katly Martins Gualberto Vaz: anakatly@ufam.edu.br

\section{Resumo}

As Infecções Relacionadas à Assistência à Saúde configuram um grande ônus orçamentário às instituições, além de prolongar o tempo de afastamento do paciente de suas atividades. Essas infecções são consideradas mais graves na Unidade de Terapia Intensiva. O presente estudo objetivou identificar as infecções prevalentes em uma Unidade de Terapia Intensiva de um hospital universitário da cidade de Manaus. Trata-se de uma pesquisa descritiva e retrospectiva, com dados do período de janeiro a dezembro de 2018. Os dados foram coletados por meio do banco de informações da Comissão de Controle de Infecção Hospitalar do hospital em estudo e analisados estatisticamente. O estudo revelou que pacientes acometidos por Infecções Relacionadas à Assistência à Saúde tiveram maior tempo de internação hospitalar. A maior prevalência foi entre pacientes cirúrgicos. A infecção do trato urinário mostrou-se a infecção mais frequente. Outro achado foi o uso prolongado de dispositivos invasivos. Considerando que essas infecções têm alto potencial preventivo, para reduzi-las os profissionais devem aderir a cuidados básicos, como a lavagem das mãos e o uso de equipamentos de proteção individual. Além disso, deve-se investir em ações de educação continuada e implementação de protocolos.

Palavras-chave: infecção hospitalar, unidades de terapia intensiva, Enfermagem.

\begin{abstract}
Prevalent infections in the intensive care unit of a university hospital

Health Care Related Infections are a large financial onus in institutions, as well as prolonging time away of the patient from his activities. These infections are considered more severe in the Intensive Care Unit. The present study aimed to identify prevalent infections in an Intensive Care Unit of a university hospital in the city of Manaus. This is a descriptive and retrospective research, with data from January to December 2018. Data were collected and analyzed through the database of the Hospital Infection Control Commission of the hospital. The study revealed that patients affected by Health Care Related Infections had longer time of hospital stay. Of these patients, most were surgical. Urinary Tract Infection was the most frequent infection. Another finding was the prolonged use of invasive devices. Considering that these infections have high preventive potential, professionals must adhere to basic care, such as hand washing and use of personal protective equipment. In addition, continuing education actions and protocol implementation must be stimulated.
\end{abstract}

Keywords: cross infection, intensive care units, Nursing. 


\section{Resumen}

Infecciones prevalentes en unidad de cuidados intensivos de un hospital universitario

La Infección Hospitalaria supone una gran carga presupuestaria a las instituciones, además de prolongar el tiempo del paciente lejos de sus actividades. Esta infección se considera una de la más grave en la Unidad de Cuidados Intensivos. El presente estudio tuvo como objetivo identificar las infecciones prevalentes en una Unidad de Cuidados Intensivos de un hospital universitario en la ciudad de Manaus. Esta es una investigación descriptiva y retrospectiva, con datos de enero a diciembre de 2018. Los datos fueron recopilados a través de la base de datos de la Comisión de Control de Infecciones Hospitalarias del hospital en estudio y analizados estadísticamente. El estudio reveló que los pacientes afectados por Infección Hospitalaria tenían estancias hospitalarias más largas. De estos pacientes, la mayoría eran quirúrgicos. La Infección del Tracto Urinario fue la infección más frecuente. Otro hallazgo fue el uso prolongado de dispositivos invasivos. Teniendo en cuenta que esta infección tiene un alto potencial preventivo, para reducirlas, los profesionales deben cumplir con los cuidados básicos, como el lavado de manos y el uso de equipos de protección personal. Además, uno debe invertir en acciones de educación continua e implementación de protocolos.

Palabras-clave: infección hospitalaria, unidades de cuidados intensivos, Enfermería.

Introdução

As Infecções Relacionadas à Assistência à Saúde (IRAS) são definidas como uma condição local ou sistêmica resultante de uma reação adversa à presença de um agente infeccioso ou sua toxina, podendo se manifestar a partir de 48 horas após a internação [1].

Existem três principais forças que estão envolvidas nessas infecções: a primeira é o uso excessivo de antimicrobianos; a segunda é a falha na adoção de medidas básicas de controle, tais como higienização das mãos, e a terceira é constituída por pacientes hospitalizados com sistema imune muito comprometido [2]. Já os principais fatores de risco relacionados às IRAS incluem, geralmente, idade superior a 60 anos, uso de corticoides, uso prévio de antibióticos, internação prolongada e presença de dispositivos médicos invasivos, tais como cateteres venosos, centrais e arteriais, diálise, ventilação mecânica e intervenções cirúrgicas [3,4].

Essas infecções se caracterizam como um problema mundial. $\mathrm{Na}$ Europa, aproximadamente $6,8 \%$ dos pacientes internados adquirem, pelo menos, uma Infecção Relacionada à Assistência à Saúde. Estima-se que nos Estados Unidos, anualmente, ocorram mais de dois milhões de casos de IRAS. No Brasil, não há dados sistematizados sobre o assunto, porém, o Ministério da Saúde considera que a taxa global de infecções gire em torno de 14\%, um número preocupante frente às consequências advindas pelas infecções [5].

Apesar das lacunas existentes nas informações, sabe-se que as IRAS se encontram entre as seis principais causas de óbito no Brasil, estando ao lado das doenças cardiovasculares, neoplasias, doenças respiratórias e infecciosas [6]. Além disso, essas infecções configuram um grande ônus orçamentário às instituições em decorrência dos custos hospitalares gerados pelo aumento na demanda das internações e, em relação ao paciente, pelo prolongamento do período de afastamento de suas atividades profissionais e familiares. O paciente que desenvolve uma Infecção Relacionada à Assistência à Saúde pode gerar um custo até três vezes maior em relação ao valor gasto com o paciente que não a desenvolveu [7].

As IRAS são consideradas mais graves na Unidade de Terapia Intensiva (UTI), em que são atendidos pacientes dependentes de suporte intensivo de vida [8]. Neste ambiente, o paciente está mais exposto ao risco de infecção, haja vista sua condição clínica e a variedade de procedimentos invasivos rotineiramente realizados. Estudo reforça que, na UTI, os pacientes têm de 5 a 10 vezes mais probabilidades de contrair infecção e que esta pode representar cerca de $20 \%$ do total das infecções de um hospital [4].

Dada a complexidade do controle das IRAS, principalmente em UTI, torna-se primordial, como ponto de partida para desenvolver medidas de prevenção e controle das infecções hospitalares, identificar as infecções prevalentes nesse setor.

Ante o exposto, este estudo objetivou identificar as infecções prevalentes em uma Unidade de Terapia Intensiva de um hospital universitário da cidade de Manaus. 
Trata-se de um estudo descritivo e retrospectivo. A pesquisa descritiva descreve as características de determinadas populações ou fenômenos, e o pesquisador deverá descobrir a frequência com que esses fenômenos acontecem $[9,10]$. Em relação ao caráter retrospectivo, o estudo é desenhado para explorar fatos do passado, podendo ser delineado para retornar, do momento atual até um determinado ponto no passado [11].

O loco da pesquisa foi um hospital universitário da cidade de Manaus, de pequeno porte, com 159 leitos [12]. Utilizaram-se os registros da Comissão de Controle de Infecção Hospitalar a respeito das infecções na UTI do hospital em estudo, no período de janeiro a dezembro de 2018.

A coleta de dados foi iniciada mediante aprovação no Comitê de Ética em Pesquisa (CEP) da Universidade Federal do Amazonas, sob o número do CAAE 07212819.6.0000.5020 e Protocolo 3.211.480, de 20 de março de 2019. Foi solicitada ao CEP a dispensa do Termo de Consentimento Livre e Esclarecido, por se tratar da análise de dados secundários, não havendo contato com os pacientes no momento da coleta dos mesmos.

Para o registro dos dados de interesse para o estudo, utilizou-se um instrumento próprio, em que constaram as seguintes variáveis: tipo de infecção, sexo, idade, diagnóstico, tempo de internação, microrganismo causador da infecção, dispositivos invasivos utilizados e desfecho da IRAS.

As informações coletadas foram reunidas em banco de dados, pelo software Microsoft Office Excelß 2007, para análise e posterior construção de tabelas e gráficos. Para facilitar a leitura dos dados, cada item foi tabulado de forma a ser analisado e contabilizado estatisticamente. A partir disso, a porcentagem de todas as IRAS foi disposta em tabelas com o intuito de quantificar os dados. Baseando-se na análise desses dados brutos, pôde-se utilizar a linguagem matemática para buscar conclusões a respeito das infecções prevalentes na UTI do hospital em estudo.

Resultados

Foram encontrados 62 casos de IRAS, em 36 pacientes. Do total de pacientes, observouse prevalência em homens, com 21 (58,33\%) casos e em idosos, com 18 (50,0\%) casos. Em relação à presença de ferida operatória, 24 (66,67\%) deles eram cirúrgicos e $12(33,33 \%)$ nãocirúrgicos. Dos pacientes cirúrgicos, 11 (30,56\%) pertenciam à especialidade da Cirurgia Geral, $10(27,78 \%)$ da Neurocirurgia, dois (5,56\%) da Cirurgia Torácica e um $(2,78 \%)$ da Ortopedia. Dos não-cirúrgicos, quatro (11,11\%) pacientes eram da especialidade da Neurologia, três $(8,33 \%)$ da Hematologia, dois (5,56\%) da Reumatologia, dois (5,56\%) da Gastroenterologia e um $(2,78 \%)$ da Nefrologia.

Em relação à presença de Lesão por Pressão (LPP), 16 (44,44\%) pacientes apresentaram registro. Durante a permanência na UTI, a maior parte dos pacientes que apresentaram IRAS, 12 (33,33\%), permaneceu internada por até 10 dias. Após a transferência para as enfermarias, o tempo de internação no hospital universitário mostrou-se um fator exponencial, com 18 (50,0\%) pacientes permanecendo internados por mais de 40 dias.

No que diz respeito à recorrência dessas infecções, 14 (38,89\%) pacientes apresentaram mais de um tipo de IRAS e 16 (44,44\%) apresentaram a mesma infecção mais de uma vez. Em relação ao desfecho do caso clínico, 20 (55,56\%) pacientes obtiveram alta hospitalar por melhora e 16 (44,44\%) evoluíram para óbito.

Dos 62 casos de IRAS, 21 (33,87\%) eram de infecção do trato urinário, $18(29,03 \%)$ de pneumonia associada à ventilação mecânica, 12 (19,35\%) de infecção de corrente sanguínea, sete $(11,30 \%)$ de infecção de sítio cirúrgico, dois $(3,22 \%)$ de infecção gastrointestinal e dois $(3,22 \%)$ de infecção do sistema nervoso central (Figura 1).

Os microrganismos mais encontrados foram Enterobacter spp. e Candida spp., com 11 $(17,74 \%)$ casos cada, seguidos de Staphylococcus spp. e Pseudomonas spp., com 10 (16,13\%) casos cada. No que concerne à relação microrganismo por tipo de IRAS, observou-se o seguinte: nos casos de infecção do trato urinário, cinco (8,06\%) foram causados por microrganismos do gênero Klebsiella spp. e cinco (8,06\%) por Candida spp.. Nos casos de pneumonia associada à ventilação mecânica, cinco $(8,06 \%)$ casos originaram-se a partir de infecção causada por bactérias Enterobacter spp. 


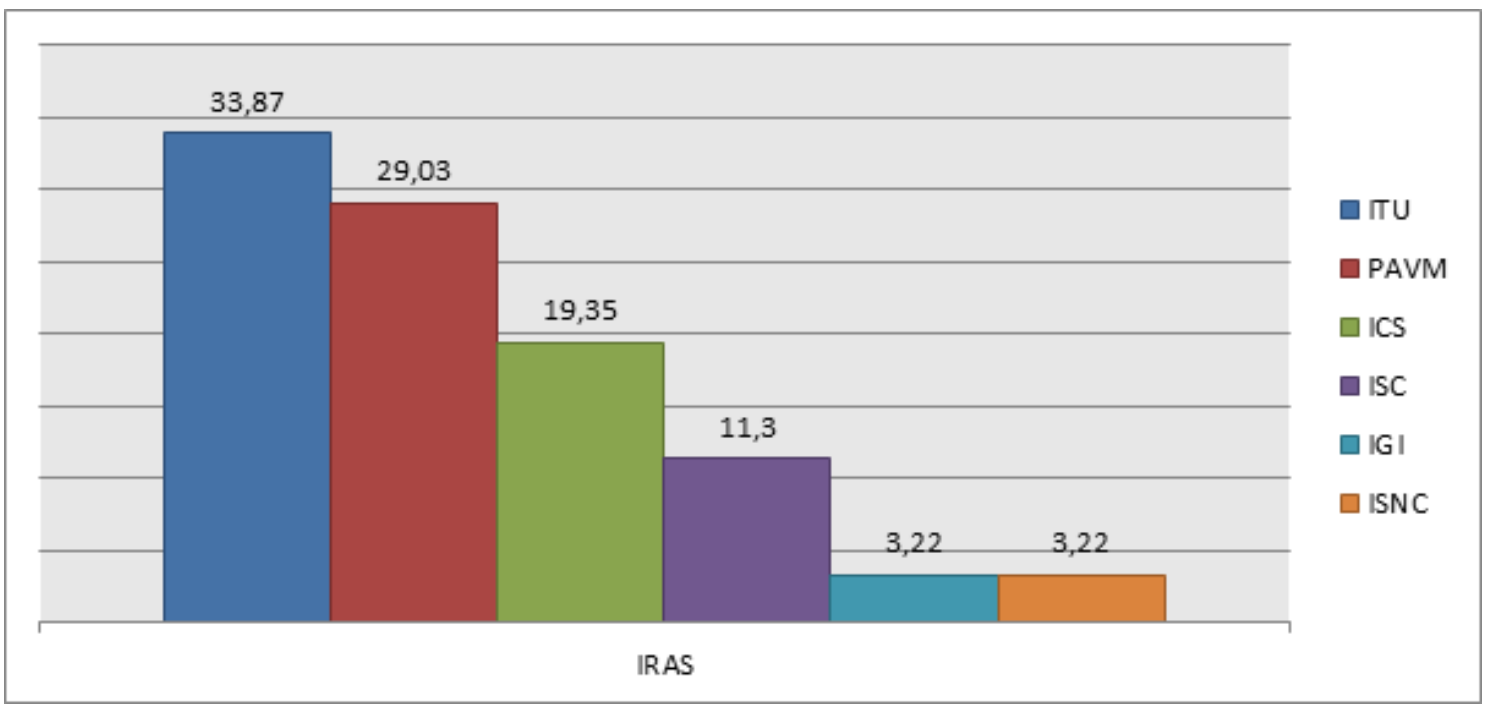

ITU = Infecção do Trato Urinário; PAVM = Pneumonia Associada à Ventilação Mecânica; ICS = Infecção de Corrente Sanguínea; ISC = Infecção de Sítio Cirúrgico; IGI = Infecção Gastrointestinal; ISNC = Infecção do Sistema Nervoso Central.

Figura 1 - Infecções Relacionadas à Assistência à Saúde notificadas na Unidade de Terapia Intensiva, segundo a área de infecção. Manaus, 2018.

As bactérias do gênero Staphylococcus spp. foram responsáveis por cinco $(8,06 \%)$ casos de infecção de corrente sanguínea. Citrobacter spp. e Staphylococcus spp., com dois $(3,22 \%)$ casos cada, originaram a maioria das infecções de sítio cirúrgico. Os dois $(3,22 \%)$ casos de infecção gastrointestinal foram causados por Enterobacter spp. e as infecções do sistema nervoso central por Pseudomonas spp. e Staphylococcus spp., com um $(1,61 \%)$ caso cada.

Em relação ao uso de dispositivos invasivos, 16 (88,89\%) casos de pneumonia associada à ventilação mecânica apresentaram tempo de uso de tubo orotraqueal maior que cinco dias, $17(80,95 \%)$ casos de infecção do trato urinário permaneceram por mais de 10 dias com cateter vesical de demora, três $(25,0 \%)$ casos de infecção de corrente sanguínea ultrapassaram 21 dias de uso de cateter venoso central e os dois $(100,0 \%)$ casos de infecção do sistema nervoso central apresentaram tempo de uso de cateter para derivação ventricular externa superior a 10 dias (Tabela I). No que diz respeito à realização de procedimentos invasivos, os dois $(100,0 \%)$ casos de infecção gastrointestinal realizaram paracentese.

Tabela I - Distribuição dos casos de infecções relacionadas à assistência à saúde na unidade de terapia intensiva, segundo o dispositivo invasivo usado e tempo de permanência. Manaus, 2018.

\begin{tabular}{ccccc}
\hline $\begin{array}{c}\text { Dispositivos } \\
\text { invasivos }\end{array}$ & IRAS & $\begin{array}{c}\text { Tempo de } \\
\text { permanência }\end{array}$ & $\mathbf{N}$ & $\%$ \\
\hline TOT & PAVM & $<5$ dias & 2 & 11,11 \\
& & $>5$ dias & 16 & 88,89 \\
\hline CVD & ITU & < d dias & 2 & 9,52 \\
& & $>10$ dias & 2 & 9,52 \\
CVC & \multirow{2}{*}{ ICS } & $<21$ dias & 9 & 80,95 \\
& & $>21$ dias & 3 & 75,00 \\
CDVE & ISNC & $>10$ dias & 2 & 25,00 \\
\hline
\end{tabular}

TOT = Tubo Orotraqueal; CVD = Cateter Vesical de Demora; CVC = Cateter Venoso Central; CDVE = Cateter para Derivação Ventricular Externa; PAVM = Pneumonia Associada à Ventilação Mecânica; ITU = Infecção do Trato Urinário; ICS = Infecção de Corrente Sanguínea; ISNC = Infecção do Sistema Nervoso Central.

\section{Discussão}

O estudo mostrou que as Infecções Relacionadas à Assistência à Saúde acometeram principalmente idosos e pacientes cirúrgicos. A idade encontrada coincide com a literatura que aponta que pacientes idosos se encontram mais suscetíveis às complicações infecciosas que acabam por estender o tempo de convalescença, devido ao processo de imunossenescência, às doenças crônico-degenerativas e a toda fisiologia do envelhecimento [13]. 
Em relação à prevalência das IRAS em pacientes cirúrgicos, estudo realizado em dois hospitais públicos do Distrito Federal mostrou que a cirurgia eletiva é um dos principais fatores de risco para a ocorrência dessas infecções no cenário de terapia intensiva. A dificuldade técnica e a duração prolongada da cirurgia, a preparação pré-operatória e a esterilização inadequada dos instrumentos cirúrgicos podem agravar o risco de infecção de forma significativa [14].

A respeito da ocorrência de lesão por pressão, sabe-se que pacientes internados em UTI apresentam alto risco para desenvolver esse tipo de lesão, devido ao uso de drogas sedativas e analgésicas, as quais diminuem a percepção sensorial e prejudicam a mobilidade [15]. No presente estudo, embora a maioria dos pacientes acometidos pelas IRAS não apresentasse LPP $(44,44 \%)$, esse valor apresenta-se elevado quando comparado à pesquisa realizada em outro hospital. Estudo realizado em um hospital universitário da Paraíba apresentou ocorrência de $22,07 \%$ desse tipo de lesão em pacientes de terapia intensiva. Esses pacientes eram, na sua maioria, mulheres idosas, com distúrbios respiratórios e presença de co-morbidades [16].

Essa diferença pode ter ocorrido devido à diferença no perfil dos pacientes avaliados na Paraíba, que eram predominantemente cirúrgicos, principalmente das especialidades de Cirurgia Geral e Neurocirurgia. Geralmente, esses pacientes são submetidos a cirurgias extensas que necessitam, muitas vezes, manter a sedação por alguns dias após o procedimento. Isso ocasiona a perda da percepção sensorial e a imobilidade, o que explica o número elevado de casos de lesão por pressão na UTI em estudo.

Observou-se também que pacientes acometidos por IRAS na UTI apresentaram maior tempo de internação no hospital. Quase a metade dos pacientes apresentou mais de uma infecção, evoluindo para óbito. Esses dados mostram que os custos dessas infecções impõem um encarecimento do atendimento, na medida em que causam aumento das demandas terapêuticas (gastos com antibióticos), da permanência hospitalar e da morbimortalidade [17].

Os pacientes que desenvolveram infecção do trato urinário apareceram em primeiro lugar nos casos de IRAS, sendo essa uma infecção de grande potencial preventivo, devido a sua relação com a cateterização vesical [18]. Esse fato demonstra a falta de intervenção educativa por meio de treinamento dos profissionais de saúde da instituição, além do estabelecimento de protocolos e bundles.

Estudo realizado em um Hospital de Alta Complexidade de Mato Grosso do Sul mostra que a educação continuada e a manutenção de protocolo apresentam efeitos favoráveis na redução de casos notificados de infecção do trato urinário [18].

Em relação aos microrganismos prevalentes na presente pesquisa, as enterobactérias, responsáveis pela maioria dos casos de pneumonia associada à ventilação mecânica, são consideradas os patógenos oportunistas mais comumente envolvidos na etiologia de pneumonia nosocomial [19].

Também foi possível observar a presença do fungo Candida spp., principalmente nas duas infecções mais encontradas, as dos tratos urinário e respiratório. Esse microrganismo é facilmente encontrado na mucosa bucal, gastrintestinal e vaginal de pessoas saudáveis, o que sugere que apenas a presença do agente etiológico não é suficiente para produzir clinicamente a doença. É necessário que ocorram modificações no estado de equilíbrio do hospedeiro, favorecendo a patogênese dos microrganismos integrantes da microbiota. Dessa forma, quando a virulência do fungo supera a resistência do hospedeiro, o fungo passa da condição saprófita para a parasitária [20].

Outros microrganismos encontrados com destaque foram as bactérias do gênero Staphylococcus spp. Essas apresentam índices de resistência significativos nos hospitais brasileiros, em torno de 40 a $80 \%$, tornando-se um problema para pacientes de terapia intensiva, devido a sua susceptibilidade ao desenvolvimento de infecções [3]. Isso justifica a presença desses patógenos na maioria dos casos de infecção de corrente sanguínea, infecção de sítio cirúrgico e infecção do sistema nervoso central encontradas neste estudo, e, em alguns casos de pneumonia associada à ventilação mecânica.

Esse fato reforça que a adesão de medidas simples e de baixo custo, como a higienização constante das mãos e o uso de equipamentos de proteção individual, podem reduzir de forma significativa o número de IRAS. Essas ações, além de diminuir as despesas hospitalares, reintegram os pacientes à vida em sociedade mais rapidamente.

As Pseudomonas spp. também apresentaram números expressivos no estudo, estando presentes nos casos de infecção do trato urinário, pneumonia associada à ventilação mecânica, infecção de corrente sanguínea e infecção do sistema nervoso central. Considerada o principal patógeno do grupo das bactérias gram-negativas, provoca infecções em pacientes com deficiência nas suas defesas antimicrobianas, causando febre, choque, oligúria, leucocitose e 
leucopenia, coagulação intravascular disseminada e síndrome da angústia respiratória no adulto [17].

Em relação ao uso de dispositivos invasivos, a Agência Nacional de Vigilância Sanitária (Anvisa) não indica rotina de troca para o tubo orotraqueal, devendo ser retirado assim que as condições clínicas permitirem [21]. Porém, estudo realizado em um Hospital de Urgências e Emergências de Sorocaba destaca que a traqueostomia precoce, realizada entre o $5^{\circ}$ e o $7^{\circ}$ dia de intubação orotraqueal, reduz a presença de infecção e, consequentemente, a mortalidade de pacientes intubados [22]. No presente estudo, a maioria dos casos de pneumonia associada à ventilação mecânica ultrapassou esse tempo.

No que concerne ao uso de cateter vesical de demora, a Anvisa também não determina rotina de troca, devendo ser trocado em caso de dano no circuito de drenagem, obstrução da sonda ou em casos de tratamento de infecção do trato urinário [21]. A presente pesquisa mostrou semelhança com estudo realizado em dois hospitais de grande porte de Belo Horizonte. Os hospitais mineiros apresentaram tempo de permanência do cateter vesical de demora maior entre os pacientes que desenvolveram infecção do trato urinário (mediana de 10 dias), quando comparados aos que não a desenvolveram (mediana de cinco dias) [23].

Em relação ao uso de cateter para derivação ventricular externa, não há rotina de troca estabelecida pela Anvisa, devendo ser retirado assim que clinicamente possível [21]. A taxa de infecção, no presente estudo, mostrou-se significativamente maior em pacientes que utilizaram esse dispositivo por mais de 10 dias. Resultado similar foi encontrado em estudo realizado em um hospital localizado em Porto Alegre, onde pacientes que utilizaram o cateter por mais de 10 dias apresentaram uma chance maior de infecção do que os pacientes que utilizaram por um tempo menor ou igual a 10 dias [24].

No que diz respeito ao uso de cateter venoso central, a Anvisa orienta que o tempo de uso do cateter de curta permanência deve ser de até 21 dias [21]. Estudo realizado em um Hospital Universitário de Uberaba destaca que a relação entre o tempo de permanência do cateter e o número total de complicações não é estatisticamente significante [25]. Resultado semelhante foi encontrado no presente estudo, visto que a maioria dos casos de infecção de corrente sanguínea ocorreu em pacientes que utilizaram o cateter venoso central por menos de 21 dias.

A despeito do impacto enorme da infecção de corrente sanguínea, essa é a infecção associada a cuidados em saúde de maior potencial preventivo que existe. Essa prevenção ocorre através da adoção de medidas adequadas, como adesão aos bundles de boas práticas de inserção e a otimização das práticas de manutenção dos dispositivos [21].

De maneira geral, a maioria dos casos de IRAS apresentam alto potencial de prevenção. Para minimizar a ocorrência de erro durante a assistência ao paciente, é necessário um processo de gestão do cuidado nos hospitais, com processos de educação continuada e implementação de protocolos, ou guia de recomendação clínica, identificando ações que ajudem a evitar danos decorrentes da assistência com o paciente [18].

Conclusão

O estudo evidenciou que as Infecções Relacionadas à Assistência à Saúde do trato urinário foram as mais encontradas dentro da UTI, seguidas do trato respiratório. Idosos e pacientes cirúrgicos caracterizaram a maioria dos casos dessas infecções. Pacientes acometidos por IRAS apresentaram maior tempo de internação hospitalar, e quase a metade deles evoluiu para óbito.

Os patógenos mais encontrados foram Enterobacter spp. e Candida spp., o primeiro foi comumente encontrado nas culturas de aspirado traqueal e, o segundo, nas culturas de urina. Em seguida, encontramos Staphylococcus spp. e Pseudomonas spp., o primeiro com prevalências nas hemoculturas e, o segundo, nas culturas de aspirado traqueal.

Em relação ao uso de dispositivos invasivos, o tempo de uso de tubo orotraqueal, cateter vesical de demora e cateter para derivação ventricular externa mostrou-se maior quando comparado a outros estudos. No que diz respeito ao uso de cateter venoso central, os pacientes que apresentaram infecção de corrente sanguínea não utilizaram o dispositivo por tempo além do preconizado.

Considerando que as IRAS são infecções com alto potencial preventivo, para reduzi-las todos os profissionais devem estar engajados nesta missão, através de cuidados básicos, como a lavagem correta das mãos e o uso de equipamentos de proteção individual. Além disso, deve- 
se investir em ações para capacitar as equipes assistenciais, como educação continuada e implementação de protocolos.

1. Silva AG, Oliveira AC. Adesão às medidas para prevenção da infecção da corrente sanguínea relacionada ao catéter venoso central. Enferm Foco 2017;8(2):36-

41. http://revista.cofen.gov.br/index.php/enfermagem/article/view/977/378

2. Torres RA, Torres BR. Importância e bases de um programa de controle e prevenção de infecção em unidade de terapia intensiva geral. Rev Med Minas Gerais (Online) 2015;25(4):577-82. Disponível em: http://rmmg.org/artigo/detalhes/1874

3. Sousa DM, Sousa AFL, Ibiapina ARS, Queiroz AAFLN, Moura MEB, Araújo TME. Infecção por Staphylococcus aureus resistente em unidades de terapia intensiva: revisão integrativa. Rev Enferm UFPE online 2016;10(4):1315-23. [citado 2018 Aug 7]. Disponível em: https://periodicos.ufpe.br/revistas/revistaenfermagem/article/viewFile/11119/12599

4. Candido TS, Bernardi ACA. Avaliação da resistência a antimicrobianos de Staphylococcuscoagulase negativa encontrados nas grades dos leitos em uma unidade de terapia intensiva. J Health Sci (Londrina) 2016;18(1):33-6. [citado 2018 Aug 6]. Disponível em:

http://www.pgsskroton.com.br/seer/index.php/JHealthSci/article/view/3513/3131

5. Ribeiro FDO, Souza MA, Paula AO, Silva AG, Oliveira AC. Estratégia lúdica para a melhoria de práticas de higienização das mãos entre os profissionais de saúde. Rev. enferm. UFPE online 2017;11(10):3971-9. [citado 2018 Aug 7]. Disponível em: https://periodicos.ufpe.br/revistas/revistaenfermagem/article/view/25207/24375

6. Michelin AF, Fonseca MRCC. Perfil epidemiológico das infecções hospitalares na unidade de terapia intensiva de um hospital terciário. Nursing (São Paulo) 2018; 21(236):2037-41. [citado 2018 Aug 6]. Disponível em:

http://www.revistanursing.com.br/revistas/236Janeiro2018/perfil epidemiologico das infeccoes hospitalares.pdf

7. Silva PLN, Aguiar ALC, Gonçalves RPF. Relação de custo-benefício na prevenção e no controle das infecções relacionadas à assistência à saúde em uma unidade de terapia intensiva neonatal. J Health Biol Sci (Online) 2017;5(2):142-9. [cited 2018 Aug 6]. Disponível em: http://periodicos.unichristus.edu.br/index.php/jhbs/article/view/1195/421

8. Perna TDGS, Puiatti MA, Perna DH, Pereira NMM, Couri MG, Ferreira CMD. Prevalência de infecção hospitalar pela bactéria do gênero Klebsiella em uma unidade de terapia intensiva. Rev Soc Bras Clín Med 2015;13(2):119-23. [citado 2018 Aug 6]. Disponível em: http://files.bvs.br/upload/S/1679-1010/2015/v13n2/a4740.pdf

9. Gil AC. Como elaborar projetos de pesquisa. $4^{\mathrm{a}}$ ed. São Paulo: Atlas; 2008.

10. Barros AJP, Lehfeld NAS. Fundamentos de metodologia científica. $3^{a}$ ed. São Paulo: Pearson Prentice Hall; 2007.

11. Hulley SB. Delineando a pesquisa clínica: uma abordagem epidemiológica. $2^{a}$ ed. Porto Alegre: Artmed Editora; 2006.

12. Universidade Federal do Amazonas [Internet]. HUGV/Ebserh receberá novos profissionais por meio de contratação via Ministério da Educação. [citado 2018 Dec 20]. Disponível em: https://ufam.edu.br/2013-04-29-19-37-05/arquivo-de-noticias/8314hugv-ebserh-recebera-novos-profissionais-por-meio-de-contratacao-via-ministerio-daeducacao

13. IzaiasEM, Dellaroza MSG, Rossaneis MA, Belei RA. Custo e caracterização de infecção hospitalar em idosos. Ciênc Saúde Coletiva 2014;19(8):3395-402. https://doi.org/10.1590/1413-81232014198.12732013

14. Sinésio MCT, Magro MCS, Carneiro TA, Silva KGN. Fatores de risco às infecções relacionadas à assistência em unidades de terapia intensiva. Cogitare Enferm 2018;23 (2): e53826. https://doi.org/10.5380/ce.v23i2.53826

15. Barbosa JM, Salomé GM. Ocorrência de lesão por pressão em pacientes internados em um hospital-escola. J Enteros Ther 2018;16(e2718):1-8. https://doi.org/10.30886/estima.v16.523 PT

16. Farias ADA, Leal NTR, Travassos NPR, Farias AJA, Nobre AMD, Almeida TCE. Ocorrência de lesões por pressão em unidade de terapia intensiva de um hospital 
universitário. Nursing (São Paulo) 2019; 22 (253): 2927-31. [citado 2020 Fev 15]. Disponível em: https://pesquisa.bvsalud.org/portal/resource/pt/biblio-1023875

17. Soares SGSC, Mascarenhas MDM, Moura LNB, Pereira AFM. Caracterização das infecções relacionadas à assistência à saúde em um hospital de ensino do Nordeste do Brasil. Rev Enferm UFPI 2017;6(2):37-43. [citado 2018 Dec 23]. Disponível em: http://www.ojs.ufpi.br/index.php/reufpi/article/viewFile/5933/pdf

18. Miranda AL, Oliveira ALL, Nacer DT, Aguiar CAM. Resultados da implementação de um protocolo sobre a incidência de infecção do trato urinário em unidade de terapia intensiva. Rev Latinoam Enferm 2016;24(e2804): 1-9. https://doi.org/10.1590/15188345.0866 .2804

19. Santos AKS, Araújo JA, Carvalho MM, Carvalho LRB, Coelho LS, Landim CAP. Perfil microbiológico das infecções hospitalares nas unidades de terapia intensiva. Rev Enferm UFPE on line 2016;10(3):1432-40. [citado 2019 Oct 19]. Disponível em: https://pdfs.semanticscholar.org/5d57/2b0528a6fd5ab6dc2b1a8b892dd14f26e512.pdf

20. Stramandinoli RT, Souza PHC, Westphalen FH, Bisinelli JC, Ignácio SA, Yurgel LS. Prevalência de candidose bucal em pacientes hospitalizados e avaliação dos fatores de risco. Rev Sul-Bras Odontol 2010;7(1):66-72. [citado 2020 Apr 12]; Disponível em: http://revodonto.bvsalud.org/pdf/rsbo/v7n1/a10v7n1.pdf

21. Brasil. Agência Nacional de Vigilância Sanitária (Anvisa). Medidas de prevenção de infecção relacionada à assistência à saúde. (Série: Segurança do Paciente e Qualidade em Serviços de Saúde; [Internet]. Brasília; 2017 [citado 2018 Nov 22]. Disponível em: http://portal.anvisa.gov.br/documents/33852/3507912/Caderno+4++Medidas+de+Preven\%C3\%A7\%C3\%A30+de+Infec\%C3\%A7\%C3\%A30+Relacionada +\%C3\%A0+Assist\%C3\%AAncia+\%C3\%A0+Sa\%C3\%BAde/a3f23dfb-2c54-4e64-881cfccf9220c373

22. Cardoso L, Simoneti FS, Camacho EC, Lucena RV, Guerra AF, Rodrigues JMS. Intubação orotraqueal prolongada e a indicação de traqueostomia. Rev Fac Cienc Med Sorocaba 2014;16(4):170-3. [citado 2019 Oct 6]. Disponível em: https://pdfs.semanticscholar.org/b3b4/d1a9bb4cac704600c09a6d34950eb115419b.pdf

23. Campos CC, Alcoforado CLGC, Franco LMC, Carvalho RLR, Ercole FF. Incidência de infecção do trato urinário relacionada ao cateterismo vesical de demora: um estudo de coorte. REME Rev Min Enferm 2016;20(973):1-7. [citado 2019 Oct 6]. Disponível em: http://www.reme.org.br/artigo/detalhes/1109

24. Santos SC. Fatores de risco para infecção relacionada à drenagem ventricular externa nas hemorragias cerebrais espontâneas em adultos. [Dissertação]. Universidade Federal do Rio Grande do Sul, 2016. [citado 2019 Oct 6]. Disponível em: https://lume.ufrgs.br/bitstream/handle/10183/150702/001008713.pdf?sequence=1\&isAll owed =y

25. Silva JAJ, Ferreira LA, Zuffi FB, Rezende MP, Mendonça GS. Breakdown of complications related to the use of central venous catheters in intensive therapy units. Biosci J 2018;34(3):810-7. [cited 2019 Oct 6]. Disponível em: http://www.seer.ufu.br/index.php/biosciencejournal/article/view/38510/22246 